\title{
A comparative study of indoor air pollution and its respiratory impacts in Delhi, India
}

\author{
P. Kulshreshtha \& M. Khare \\ Department of Civil Engineering, Indian Institute of Technology, India
}

\begin{abstract}
The present study aims at investigating the indoor air quality (IAQ) in selected households in the urban slums, as well as a control site, in Delhi, the capital city of India. The study includes assessments of associated respiratory health effects and Sick Building Syndrome (SBS) on the occupants living in inefficiently designed houses with poor ventilation. The comparative analysis of concentrations of indoor air pollutants in urban slums and the control site reveals that the respiratory health of the occupants living indoors in both the sites are affected by the indoor pollutants, e.g. the respirable suspended particulate matter $\left(\mathrm{PM}_{10}\right.$ and $\left.\mathrm{PM}_{2.5}\right)$, the carbon monoxide $(\mathrm{CO})$, the sulphur dioxide $\left(\mathrm{SO}_{2}\right)$ and the nitrogen dioxide $\left(\mathrm{NO}_{2}\right)$. In addition, the spirometry tests on the occupants, particularly the womenfolk and children, have been performed to determine the incidence of acute respiratory infections (ARI). Interview surveys have also been conducted in the households during the study period to investigate sick building syndrome (SBS). The results show the maximum concentration of indoor air pollutants in households during winters associated with aggravated respiratory problems, such as coughs, phlegm, wheezing, and breathlessness, among occupants, particularly the women. In addition, decrement in lung function indices (i.e. FVC and/or FEV F $_{1}$ ) due to increased concentrations of RSPM indoors during the winter period has also been observed in the women occupants. The study concludes that women and children indoors are most vulnerable to respiratory problems compared to men. Along with a higher prevalence of respiratory symptoms, high SBS scores have been observed in these household because of inefficient ventilation, poorly designed houses and usage of biomass fuels for cooking.
\end{abstract}

Keywords: indoor air quality, sick building syndrome, acute respiratory infections, spirometry, biomass fuel. 


\section{Introduction}

Whilst chimneys first began to appear in European homes in the late twelfth century, most large medieval houses still had a central hearth in the great hall, ventilated by a louver in the roof. It was only during the sixteenth century that chimneystacks came into general use (Burr [4]). Since as early as 1968 (Cleary and Blackburn [6]; Sofoluwe [16]), claims have been made on the effect of indoor air pollution on the health of people in developing countries. According to Bruce et al. [5], around half of the population in developing countries, and $90 \%$ of the rural population, rely on coal and biomass (wood, dung, crop residues) for domestic energy, often using simple stoves that produce incomplete combustion.

Bruce et al. [5] estimate that indoor air pollution from biomass and coal smoke is responsible for approximately 2 million annual deaths, representing around $4 \%$ of the global disease burden, with acute respiratory infections (ARIs) being the greatest cause of mortality in children under five. India has among the largest burden of disease due to the use of dirty household fuels and $28 \%$ of all deaths due to indoor air pollution occur in India (Smith [15]). Padmavati [11] has described the relationship between exposure to indoor air pollutants and chronic obstructive lung disease leading to chronic cor pulmonale. Association between use of biomass fuels and pulmonary tuberculosis has been reported indicating that amongst persons aged 20 years and above, $51 \%$ of the prevalence of active tuberculosis has been attributed to smoke from cooking fuel (Mishra et al. [9]).

In poor households in the Southern India, women typically do most of the cooking and spend most time indoors, thus they are subjected to high levels of pollution both from being close to the fire, and spending longer periods of exposure in the indoor environment (Sankar et al. [12]).

The present study aims at comparing the concentrations of indoor air pollutants in residential dwellings in commercial and non commercial areas in Delhi city and assessing its effect on the respiratory health of the occupants.

\section{Methods and materials}

\subsection{Locale of the study}

Population growth and fast developing economic activities have led to increase in ambient and indoor pollution in Delhi. This study aims at assessing the indoor air quality in the households located near the commercial areas in Delhi city.

\subsection{Selection of sample areas}

There are more than 1000 urban slum clusters in Delhi housing more than 1.5 million people (Sharma et al. [13]). One of the sampling sites for the present study is Nizamuddin area where the level of commercial activities is very high. It has one of the busiest railway stations and commercial markets. It has residential facilities belonging to 'high' [Annual income >INR. 96,000 (US\$ 2300)], 
'middle' [Annual income between INR 45,000 and 62,000 (US\$ 1070-1550)] and 'low' [Annual income <INR.22, 500 (US\$ 535)] income groups of people. For experimental work, five houses have been selected from the site i.e. three from low income group, one from middle income group and one from high income group, based on the distance from the commercial area. The first low income group household is closest to the railway station; the second is slightly far and the third household is near to the road.

I.I.T. Delhi campus has been taken as one of the sampling sites for present study as control site because there is minimum commercial activities. The site selection criteria involves existence of different stratums of population based on the socioeconomic status i.e., 'high', 'middle' and 'low' income groups. In I.I.T. Delhi campus, total 3 houses are selected for the experimental part i.e. one each from low, middle, and high income groups.

\subsection{Monitoring of pollutants}

Since the study focus is on assessment of indoor air quality of the household kitchens, it was considered that the data collection technique would not be confined to descriptive data collection alone, but would also be supplemented by experimental data. The experiments are conducted in selected household kitchens to measure the concentrations of respirable suspended particulate matter (RSPM i.e. $\mathrm{PM}_{10}$ and $\mathrm{PM}_{2.5)}$, carbon monoxide $(\mathrm{CO})$ and oxides of sulphur and nitrogen $\left(\mathrm{SO}_{2}\right.$ and $\left.\mathrm{NO}_{\mathrm{x}}\right)$. The sampling time ranges between 10 a.m. to 4 p.m. During this time cooking activity is carried out at least once in all the representative households. During weekdays, monitoring is carried on Monday, Wednesday and Friday in each household. To assess the seasonal variations of the concentration of indoor pollutants, monitoring has also been carried out in different seasons of the year i.e. April to June (summer season), and December to February (winter season). RSPM $\left(\mathrm{PM}_{10}\right.$ and $\left.\mathrm{PM}_{2.5}\right)$ both outdoors and indoors are monitored using GRIMM made Environmental Dust Monitor model 107 AEROSOL Technik GmbH \& Co KG, Dorfstrasse 9, D-83404-Ainring, Germany. APM820 (Envirotech, 2004) handy sampler for indoor and outdoor has been used for measuring $\mathrm{SO}_{2}$ and $\mathrm{NO}_{\mathrm{x}}$. The standard West-Geake spectrophotometer (IS: 5182(Part II), 1973) and the modified Jacob and Hoecheiser's (IS: 5182(Part IV), 1975) method have been used for $\mathrm{SO}_{2}$ and $\mathrm{NO}_{2}$ analysis, respectively. $\mathrm{CO}_{2}$ and $\mathrm{CO}$ has been monitored using IAQ monitor Quest-5001 (Quest Technologies, Wisconsin, USA, 2000).

\subsection{Lung function test}

Forced vital capacity (FVC), forced expiratory volume in $1 \mathrm{~s}$ (FEV1) and peak expiratory flow rate (PEFR) have been measured by spirometry. Spirometry is done with the subject in a sitting position and wearing a nose-clip under the supervision of occupational and health specialists from LNJP Hospital, Delhi (American Thoracic Society [1]). 


\subsection{Sick building syndrome scale and respiratory problem scale}

Interview surveys have been carried out to gather information on type of kitchen, type of fuel used, frequency and severity of respiratory symptoms experienced by the occupants in order to estimate the sick building syndrome (SBS). A scale of $0-15$ has been used to estimate the SBS score (Kulshreshtha et al. [8]). The Medical Research Council Committee respiratory problem scale has been used (MRC [10]) for the spirometry analysis. Student's $t$ and two Paired t-tests are used to find out pollutant concentration variations in different seasons. Pearson's and Spearman's correlations have been used for evaluating the effects of demographic variables on respiratory problem score (Kulshreshtha et al. [8]).

\section{Results and discussions}

\subsection{Mean concentrations and seasonal variation of indoor air pollutants}

In winters, the $\mathrm{PM}_{10}$ concentrations are towards higher side in almost all the households in the sampling site area. Besides, the $\mathrm{PM}_{10}$ and $\mathrm{PM}_{2.5}$ concentrations are much higher in sample site than at control site.

However, at the control site, the MIG and HIG households are showing increasing trend in the RSPM concentrations. The $\mathrm{PM}_{2.5}$ concentrations are $625.44 \mu \mathrm{g} / \mathrm{m}^{3}$ in sample site during winters; whereas the maximum concentration observed at control site is $213.72 \mu \mathrm{g} / \mathrm{m}^{3}$ (Fig. 2) It may be due to the fact that in sample site there are indoor sources like cooking activities, inadequate ventilation, higher occupancy along with penetration of outdoor air indoors. At the control site, the primary cause of higher RSPM concentrations is infiltration through openings in the households that results into increases in $\mathrm{PM}_{10}$.

The CO concentrations increase during summers (Fig. 3). In sample site, the $\mathrm{CO}$ concentrations levels are high due to vehicular exhausts and emissions from the railway station. At the control site, i.e. at IIT Delhi campus, free passage to these vehicles is restricted.

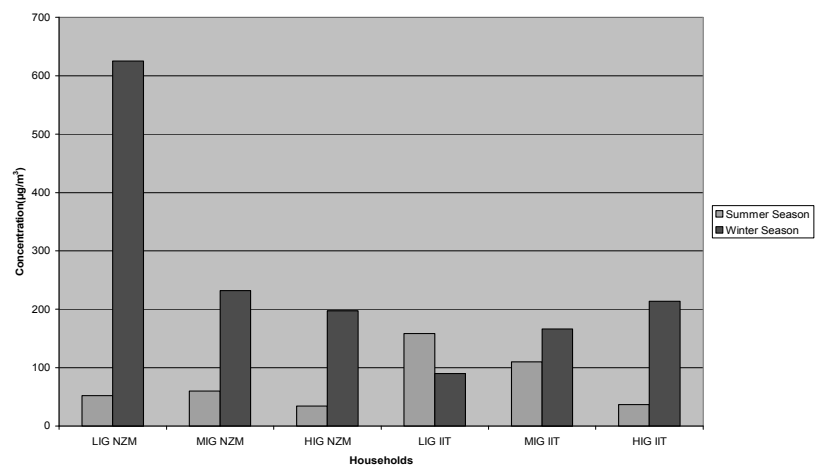

Figure 1: Mean $\mathrm{PM}_{10}$ concentration in different seasons. 


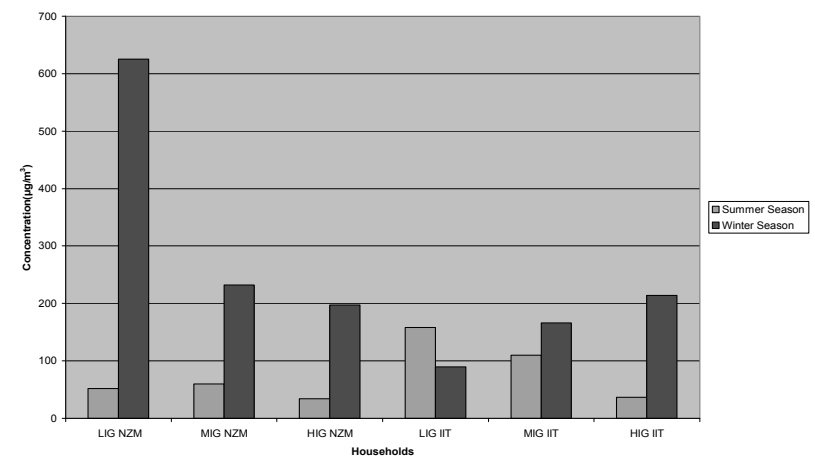

Figure 2: $\quad$ Mean concentration of $\mathrm{PM}_{2.5}$ in different seasons.

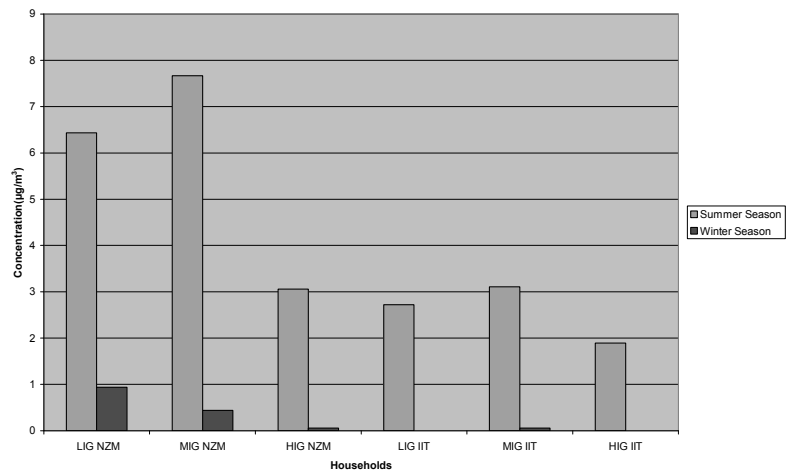

Figure 3: Mean CO concentration in different seasons.

The I.I.T. Delhi campus is much greener area with lot of open spaces, trees, and plants that help to reduce the concentrations of pollutants to a considerable extent. The concentrations of $\mathrm{SO}_{2}$ are towards higher side in LIG households at the sample site area during summers (Fig 4). In MIG and HIG households at sample site and in all households at I.I.T. Delhi campus, the $\mathrm{SO}_{2}$ is well within the permissible limits (ASHRAE [2]). The LIG occupants are using kerosene stoves or poorly vented gas appliances. It causes high $\mathrm{SO}_{2}$ concentrations indoors. No significant seasonal variations in the $\mathrm{SO}_{2}$ concentrations have been observed.

The concentrations of NOx are higher in LIG and MIG households in sample site during summers (Fig 5). Usage of wood and kerosene stoves is prevalent in these households accompanied by infiltration of outdoor pollutants indoors. At the control site, the NOx concentrations are within the permissible limits (ASHRAE [2]). 


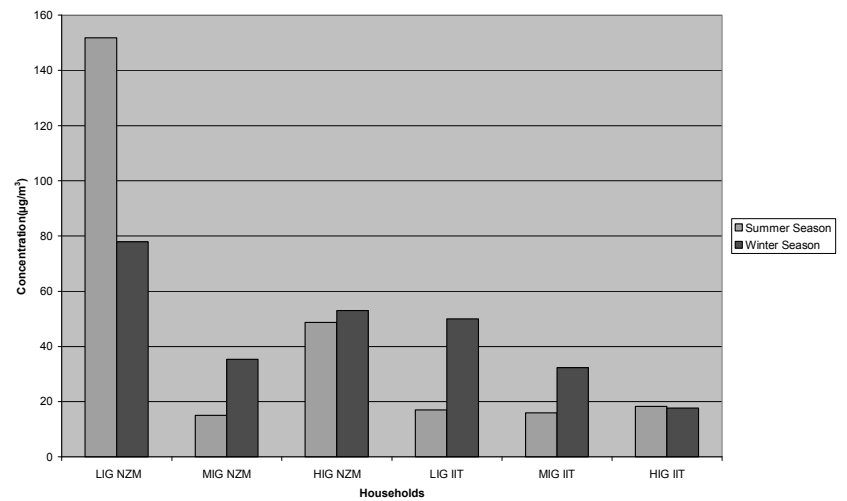

Figure 4: $\quad$ Mean $\mathrm{SO}_{2}$ concentration in different seasons.

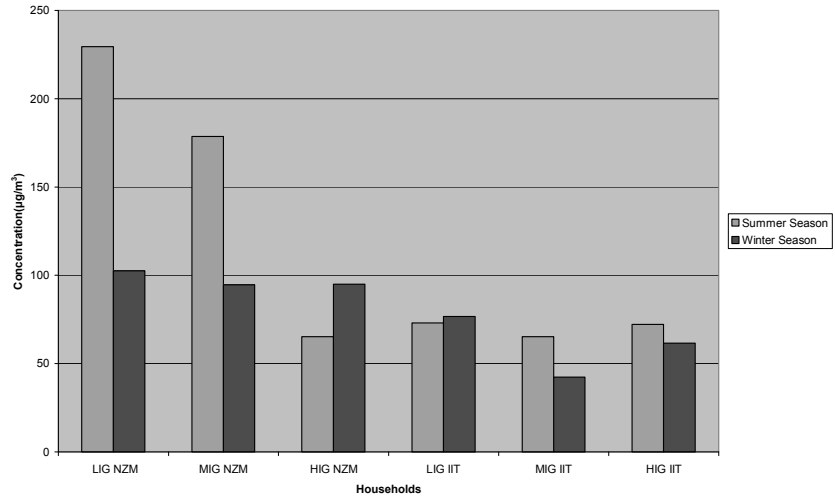

Figure 5: $\quad$ Mean $\mathrm{NO}_{\mathrm{x}}$ concentration in different seasons.

\subsection{Indoor air pollution and respiratory health}

Lung function test is a tool for evaluating respiratory status. Table 1 displays pulmonary function test in occupants from sample and control sites. The test is performed to compare expected and observed values against individual pulmonary function test amongst the occupants. The FVC in LIG occupants at sample site is 1.221 (< normal value of 2.37 1). At sample site, amongst MIG household occupants too, FVC and PEFR are below the expected normal values e.g. 1.601 (expected normal is 2.581 ) and 1.741 (expected normal is 5.991 ), respectively. Higher RSPM concentrations indoors results in reduction of FEV1 and FVC (Table 2). As a result, occupants report moderate to severe and very severe obstructions of lungs (mean FEV1 and FVC <normal value). Similar results have been obtained among occupants of MIG type households. However, among HIG household occupants, the FEV1 and FVC are normal (Table 1). 
Table 1: $\quad$ Lung function test of occupants at both sites.

\begin{tabular}{|c|c|c|c|c|c|}
\hline & \multirow{2}{*}{$\begin{array}{l}\text { Pulmonary } \\
\text { Function } \\
\text { Test(PFT) }\end{array}$} & \multicolumn{2}{|c|}{$\begin{array}{c}\text { Sample site } \\
\mathrm{n}=30\end{array}$} & \multicolumn{2}{|c|}{$\begin{array}{c}\text { Control site } \\
\mathrm{n}=30\end{array}$} \\
\hline & & Observed & Expected & Observed & Expected \\
\hline \multirow[t]{3}{*}{ LIG } & FVC(L) & $1.22 \pm 0.50$ & $2.37 \pm 0.25$ & $3.25 \pm 0.12$ & $2.44 \pm 0.51$ \\
\hline & FEV1(L) & $0.64 \pm 0.43$ & $2.04 \pm 0.21$ & $3.19 \pm 0.04$ & $2.04 \pm 0.50$ \\
\hline & PEFR(L/S) & $0.96 \pm 0.78$ & $5.67 \pm 0.96$ & $9.05 \pm 0.81$ & $6.72 \pm 0.67$ \\
\hline \multirow[t]{3}{*}{ MIG } & $\mathrm{FVC}(\mathrm{L})$ & $1.60 \pm 0.29$ & $2.58 \pm 0.45$ & $1.26 \pm 0.38$ & $2.04 \pm 0.56$ \\
\hline & FEV1(L) & $0.96 \pm 0.66$ & $2.21 \pm 0.44$ & $1.24 \pm 0.36$ & $1.82 \pm 0.53$ \\
\hline & PEFR(L/S) & $1.74 \pm 1.86$ & $5.95 \pm 0.16$ & $2.11 \pm 0.33$ & $4.02 \pm 1.64$ \\
\hline \multirow[t]{3}{*}{ HIG } & FVC(L) & $1.94 \pm 0.79$ & $2.26 \pm 0.80$ & $1.41 \pm 0.06$ & $2.01 \pm 0.39$ \\
\hline & FEV1(L) & $1.90 \pm 0.74$ & $1.92 \pm 0.79$ & $1.38 \pm 0.04$ & $1.68 \pm 0.24$ \\
\hline & PEFR(L/S) & $3.26 \pm 0.51$ & $5.91 \pm 0.21$ & $3.16 \pm 0.64$ & $6.18 \pm 0.46$ \\
\hline
\end{tabular}

Table 2: $\quad$ Effect of indoor air pollutants on the lung function test at both sites $(n=60)$.

\begin{tabular}{|c|c|c|c|c|}
\hline Household & \multicolumn{2}{|c|}{$\begin{array}{c}\text { Pollutant } \\
\text { concentrations }\end{array}$} & \multicolumn{2}{c|}{$\begin{array}{c}\text { Lung Function } \\
\text { Parameters }\end{array}$} \\
\hline & $\begin{array}{c}\text { RSPM } \\
\text { Mean } \\
\left(\boldsymbol{\mu g} / \mathbf{m}^{3}\right)\end{array}$ & $\begin{array}{c}\text { CO } \\
\text { Mean } \\
(\mathbf{p p m})\end{array}$ & $\begin{array}{c}\text { FEV1 } \\
\text { Mean }\end{array}$ & $\begin{array}{c}\text { FVC } \\
\text { Mean }\end{array}$ \\
\hline Sample site & & & & \\
\hline LIG & 338.7 & 4.0 & 0.64 & 1.22 \\
\hline MIG & 165.2 & 5.1 & 0.96 & 1.60 \\
\hline HIG & 196.7 & 3.0 & 1.90 & 1.94 \\
\hline Control site & & & & \\
\hline LIG & 148.9 & 1.4 & 3.19 & 3.35 \\
\hline MIG & 216.5 & 1.5 & 1.24 & 1.26 \\
\hline HIG & 177.8 & 0.9 & 1.38 & 1.41 \\
\hline
\end{tabular}

At the control site, the observed values for FEV1 and FVC of the occupants are within the range. Thus the lung function test reveals that the pulmonary ability of the occupants at control site has been much improved than at the sample site.

Further, Pearson's and Spearman's correlations have been used to find correlation between age and respiratory problems, respiratory problems and gender (female population only) and education and occupation. The correlation coefficient value of -0.58 shows a high degree of negative relationship between women and respiratory problem score at the sample site. Similarly, at the control site, correlation coefficient values $(-0.37)$ at LIG and (-0.54) at HIG show a moderate negative relationship between gender and respiratory problem scores. Hence, the female population is more vulnerable to the respiratory problems amongst the occupants at both sites. 


\subsection{Indoor air pollution and sick building syndrome}

SBS questionnaires have been used and analyzed to evaluate SBS score in all household types. A SBS scale is formed consisting of 16 symptoms as described below:

$0-5=$ Low SBS score;

6-10 = Medium SBS score;

11-16 $=$ High SBS score.

In LIG and MIG type households, $56.7 \%$ of respondents have high SBS score; whereas $43.3 \%$ of respondents report, medium SBS score at the sample site (Table 3); corresponding FEV1 and FVC values are 0.64 and1.22 for LIG; and 0.96 and 1.60 for MIG (Table 1), indicating higher impairment of lungs in the occupants of LIG and MIG type of households. These houses are quite old and poorly maintained. The ventilation is also inadequate as the respondents keep their windows and doors closed most of the time. Similarly, in HIG households, use of air conditioners restricts fresh air intake. It deteriorates the IAQ and makes the households 'sick' (SBS $=11-15$ and corresponding FEV1 = 0.64 and $\mathrm{FVC}=1.22$ ).

The majority of the respondents at the sample site complain of high SBS symptoms due to inadequate ventilation, high occupancy level, and improper maintenance of appliances and ignorance of the respondents about proper ventilation. A negative relationship existed between the SBS score and the income level of the respondents. The higher the income level, the lower the SBS score. Thereby, showing that as the income level increases, the awareness regarding health issues also increases.

Table 3: $\quad$ Effect of concentration of pollutants on SBS score.

\begin{tabular}{|c|c|c|c|c|c|c|}
\hline & \multicolumn{2}{|c|}{ SBS Score } & \multicolumn{3}{c|}{ Concentration of indoor air pollutants } \\
\hline & $\begin{array}{c}\text { Medium } \\
(\mathbf{6 - 1 0 )}\end{array}$ & $\begin{array}{c}\text { High } \\
(\mathbf{1 1 - 1 5})\end{array}$ & $\begin{array}{c}\text { RSPM } \\
\left(\boldsymbol{\mu g} / \mathbf{m}^{\mathbf{3}}\right)\end{array}$ & $\begin{array}{c}\mathbf{C O} \\
(\mathbf{p p m})\end{array}$ & $\begin{array}{c}\mathbf{S O}_{\mathbf{2}} \\
\left(\boldsymbol{\mu g} / \mathbf{m}^{\mathbf{3}}\right)\end{array}$ & $\begin{array}{c}\mathbf{N O}_{\mathbf{x}} \\
\left(\boldsymbol{\mu g} / \mathbf{m}^{\mathbf{3}}\right)\end{array}$ \\
\hline $\begin{array}{c}\text { Sample } \\
\text { Site }\end{array}$ & & $\mathbf{\%}$ & & & & \\
\hline LIG & 43 & 57 & 339 & 4.0 & 152.5 & 162.8 \\
\hline MIG & 47 & 53 & 165 & 5.1 & 82.7 & 125.4 \\
\hline HIG & 47 & 53 & 196 & 3.0 & 104.5 & 86.1 \\
\hline $\begin{array}{c}\text { Control } \\
\text { Site }\end{array}$ & & & & & & \\
\hline LIG & 50 & 50 & 149 & 1.4 & 33.5 & 74.8 \\
\hline MIG & 63 & 37 & 216 & 1.5 & 24.2 & 53.8 \\
\hline HIG & 77 & 23 & 178 & 0.9 & 18 & 67 \\
\hline
\end{tabular}




\section{Conclusions}

The RSPM concentrations indoors at the sample site remain $200 \mu \mathrm{g} / \mathrm{m}^{3}$ or more during winters. However, during summers, it reduces due to efficient dispersion. The CO concentrations show small variations during summers. The NOx concentrations are towards higher side due to usage of gas and kerosene stoves indoors and penetration of outdoor air laden with vehicular exhausts.

Gender is found to be a vulnerable factor with regard to the SBS score as well as respiratory problem score at both the sites. The womenfolk at the sample site complain of more symptoms relating to their respiratory health. The lung function test results also reveals that the lung ability is poor in women at the sample site when compared with the control site. The reason for this may be due to usage of biomass fuels, inadequate ventilation and old and improperly maintained houses. This finding also lends support to other studies (Bruce et al. [5]; Colbeck et al. [7]; Siddiqui et al. [14]; Balakrishnan et al. [3]) that link household fuel use with adverse health outcomes. Certain public health policy implications such as improved stoves with chimneys, use of cleaner fuels like piped compressed natural gas, better housing and kitchen design with improved ventilation may certainly help in controlling indoor air pollution.

\section{References}

[1] American Thoracic Society, Standardization of Spirometry-1994 update, American Journal of Respiratory Critical Care Medicine, 152, pp.11071136.

[2] ASHRAE(American Society of Heating, Refrigerating and Air conditioning Engineers)Ventilation for acceptable indoor air quality ASHRAE standard 62-1989,Atlanta,American Society for heating, refrigerating and Air conditioning Engineers, 1989.

[3] Balakrishnan,K.,Parikh,J.,Sankar,S.,Padmavathi,R.,Srividya,K.,Venugopal, V.,Prasad,S and Pandey, V., Daily average exposures to respirable particulate matter from combustion of biomass fuels in rural households of southern India, Environmental Health Perspectives,110(11),pp.1069-1075.

[4] Burr, M. L., Health effects of indoor combustion products. Journal of the Royal society of Health, 117(6), pp.348-350, 1997.

[5] Bruce, N., R. Perez-Padilla and R. Albalak, Indoor air pollution in developing countries: a major environmental and public health challenge. Bulletin of the World Health Organization, 78(9), pp.1078-1092, 2000.

[6] Cleary, G. J. and Blackburn, C.R.B., Air pollution in native huts in the highlands of New Guinea. Archives of Environmental Health, 17,pp.785794,1968

[7] Colbeck, I., Nasir, Z. A. and Ali, Z., Characteristics of indoor/outdoor particulate pollution in urban and rural residential environment of Pakistan. Indoor Air, 20, pp.40-51, 2010. 
[8] Kulshreshtha, P., Seetharaman, P.S., and Khare, M., Indoor air quality assessment in and around urban slums of Delhi city, India. Indoor Air, 18, pp.488-498, 2008.

[9] Mishra, V.K., Retherford, R.D and Smith, K.R., Biomass cooking fuels and prevalence of tuberculosis in India. International Journal of Infectious diseases, 3, pp.119-129, 1999.

[10] Medical Research Council, Questionnaire on Respiratory symptoms, 21 Park Crescent, London WINS 4AL Publications Group, Medical Research Council, 1986.

[11] Padmavati, S. and Pathak, S.N., Chronic cor pulmonale in Delhi Circulation, 20, p.343.

[12] Sankar, S., Padmavathi, R. and Balakrishnan, K., Exposure assessment for indoor air pollution associated with household fuel use in rural districts of southern India-Results of monitoring exercises in Tamil Nadu and Andhra Pradesh, Proceedings of Indoor Air,pp. 3635-3639.

[13] Sharma, S., Sethi, G.S., Rohtagi, A., Chaudhary, A., Shankar, R., Bapna, J.S., Joshi, V., and Sapir, D.G., Indoor air quality and acute lower respiratory infection in Indian urban slums, Environmental Health Perspectives, 106, pp.291-297.

[14] Siddiqui, A.R., Lee, K., Bennett, D., Yang, X., Brown, K.H., Bhutta, Z.A. and Gold, E.B., Indoor carbon monoxide and PM2.5 concentrations by cooking fuels in Pakistan, Indoor Air,19,pp.75-82,2009.

[15] Smith, K.R., Indoor air pollution implicated in alarming health problems, In: Indoor Air Pollution-Energy and health for poor. Newsletter published by World Bank, p.1,2000

[16] Sofoluwe, G.O., Smoke pollution in dwellings of infants with bronchopnuemonia, Archives of Environmental health, 16, pp. 670-672, 1968. 